\title{
Mensuração de externalidades do transporte de carga brasileiro
}

[Measurement of externalities of freight transport in Brazil]

\author{
Newton de Castro* \\ Universidade Federal do Rio de Janeiro (UFRJ), Brazil
}

Submitted 25 Sep 2011; received in revised form 3 Jan 2012; accepted 26 Jan 2012

\begin{abstract}
Resumo
Neste artigo revisitam-se algumas das principais metodologias disponíveis para a estimação do custo associado às externalidades causadas pelo transporte. 0 foco é no transporte rodoviário de carga, mas os métodos elencados podem ser estendidos a outros segmentos ou modais, com as devidas adaptações. Nesse sentido, identificam-se as variáveis determinantes das externalidades ambientais e dos custos decorrentes, particularmente os relativos à poluição do ar, aos custos de congestionamento e acidentes, e ao uso da infraestrutura, bem como examinam-se as possibilidades de aplicação dos métodos propostos ao caso brasileiro.
\end{abstract}

Palavras-Chave: externalidades; transporte de carga; Brasil.

\section{Abstract}

This article reviews methodologies used in the estimation of costs imposed by externalities produced on the movement of people and freight. The focus is on freight transport by truck, although the methods selected may be extended to other modes. The determinants of externalities and their associated costs are identified, particularly those caused by air pollution, noise, accidents, and use of infrastructure. The relevance and requirements for the application of these measurements in the Brazilian context are also discussed.

Key words: externalities; cargo; Brazil.

*Email:nrdc@br.inter.net.

\section{Recommended Citation}

Castro, N. (2013) Mensuração de externalidades do transporte de carga brasileiro. Journal of Transport Literature, vol. 7, n. 1, pp. 163-181.

- JTL/RELIT is a fully electronic, peer-reviewed, open access, international journal focused on emerging transport markets and published by BPTS - Brazilian Transport Planning Society. Website www.transport-literature.org. ISSN 2238-1031. 


\section{Introdução}

Desde os primeiros registros conhecidos da história da humanidade, o desenvolvimento dos meios de transporte tem sido um dos principais propulsores do crescimento econômico. Os exemplos percorrem o tempo: da domesticação de animais para monta ou tração, passando pela invenção da roda, os sistemas de rodovias do Império Romano, o domínio da navegação em alto mar, e chegando às ferrovias e ao motor à explosão no século XIX, e à aviação no início do século passado.

Ao final do século XX, no entanto, a crença de que a expansão da oferta de transporte e aumento da mobilidade é um bem incontestável começa a ser questionada. De fato, de um lado a ampla oferta de meios de transporte atenuou fortemente o custo de deslocamento, de outro, a crescente demanda de viagens produziu os congestionamentos, poluição e acidentes. Os custos sociais do transporte passaram pesar de forma significativa sobre a sociedade, com uma distribuição muitas vezes iníqua. ${ }^{1}$

No Brasil, a frota de veículos automotores cresceu de 18 para 64 milhões, entre 1990 e 2010, mesmo com crescimento tímido da economia observado até o início dos anos 2000. A permanecer esse padrão de evolução, chegaremos a 2030 com um frota próxima de 230 milhões de veículos. Claramente, um padrão não sustentável de evolução. Ademais, os transportes, em geral, consomem cerca de $28 \%$ da energia total do país, sendo que $92 \%$ desse consumo se dá no transporte rodoviário. Dos derivados de petróleo consumidos no país, o setor de transportes é responsável por $51 \%$.

Assim, o interesse de avaliar os custos sociais e as externalidades do transporte, em geral, vem crescendo de forma significativa, nas últimas décadas, atendendo ao reclame da sociedade por políticas mais eficazes de controle dos efeitos negativos associados ao transporte, em particular o rodoviário. Com efeito, diversos programas de redução de acidentes, congestionamento e poluição do ar vêm sendo implementados no Brasil e no mundo, tais como a regulamentação direta das emissões de veículos produzidos, as vistorias anuais dos veículos em operação - havendo interesse de se avaliar a evolução dos indicadores

\footnotetext{
${ }^{1}$ Para uma reflexão mais extensa nessa linha veja Santos, 2010.
} 
desses custos, especialmente em resposta a esses programas de controle. Avanços nesse domínio propiciariam ainda responder, ao menos parcialmente, a possibilidade do uso de políticas alternativas de preço, investimento e regulação, visando à redução dos impactos externos do transporte.

A abrangência dos impactos sociais e externos do transporte é proporcional a importância do setor para a economia em geral. Poluição do ar e sonora, congestionamento, acidentes, poluição das águas pelos resíduos deixados nas estradas e vazamentos em postos de abastecimento, disposição de resíduos sólidos, como óleos lubrificantes e pneus, destruição e perda de áreas naturais pela construção de vias, e efeito estufa causado pelos gases emitidos pelos veículos, são elementos de destaque dessa lista.

Nesse sentido, este artigo busca examinar metodologias propostas para a estimação do custo associado às externalidades causadas pelo transporte. O foco é no transporte rodoviário de carga, mas os métodos elencados podem ser estendidos a outros segmentos ou modais, com as devidas adaptações. Nesse sentido, identificam-se as variáveis determinantes das externalidades ambientais e dos custos decorrentes, particularmente os relativos à poluição do ar, aos custos de congestionamento e acidentes, e ao uso da infra-estrutura, bem como examinam-se as possibilidades de aplicação dos métodos propostos ao caso brasileiro.

Na seção seguinte alinham-se algumas medidas selecionadas do impacto agregado dessas externalidades, bem como a distribuição de sua origem por classe de veículo e de seu efeito sobre usuários e não usuários. Em seguida, tratam-se separadamente a poluição do ar, os acidentes, os congestionamentos, e os impactos sobre a infraestrutura.

\section{Medidas Selecionadas de Custos de Externalidades do Transporte}

Não obstante a importância desse tema, os instrumentos disponíveis para a quantificação dos impactos de externalidades causadas pelo transporte ainda estão longe de satisfatórios. Há controvérsias sobre a relação entre o transporte e seus impactos, bem como sobre como calcular os custos associados. Por exemplo, em 1995, o Bureau of Transportation Statistics promoveu uma conferência sobre os custos sociais do transporte, nos EUA, que projetou os 
custos sociais do transporte rodoviário, em 2000, variando entre US\$ 138 bilhões e US\$ 1 trilhão. A tabela 1 resume esses resultados, permitindo ainda discriminar a importância relativa dos principais efeitos quantificados, assim como a dificuldade apontada, na época, de quantificação de certos itens de custo (e.g., poluição do ar e outros). Observe-se a dominância dos custos sociais de acidentes (81 a 87\%) sobre os demais, para todas as classes de estimativas, seguidas de longe pelo custo de congestionamento (12 a 16\%).

\begin{tabular}{|l|r|r|r|}
\hline \multicolumn{2}{|c|}{$\begin{array}{r}\text { Tabela 1- Estimativas do Custo Social de Transporte Rodoviário nos EUA, } \\
\text { em } 2000 \\
\text { (US\$ 10^6) }\end{array}$} \\
\hline Estimativa & \multicolumn{1}{|c|}{ Alta } & \multicolumn{1}{|c|}{ Média } & \multicolumn{1}{c|}{ Baixa } \\
\hline Congestionamento & 181.635 & 61.761 & 16.352 \\
\hline Acidentes & 839.463 & 339.886 & 120.580 \\
\hline Poluição do Ar & & & \\
\hline Poluição Sonora & & & \\
\hline Total & 11.446 & & 1.214 \\
\hline
\end{tabular}

** não foi possível mensurar na ocasião. Fonte: Transportation Statistics Annual Report - BTS 1996.

A tabela 2 apresenta a abertura dos custos sociais de acordo com o tipo de veículo responsável. Face à imensa frota de automóveis e camionetes existentes nos EUA, estes são responsáveis por cerca de $93 \%$ dos custos sociais. Outro aspecto a destacar é que, excluindose os custos de poluição do ar, quase $90 \%$ dos custos sociais do transporte rodoviário são suportados, pelo menos em primeira instância pelos próprios usuários, incluindo US\$ 300 bilhões dos custos de acidentes e US\$ 60 bilhões dos custos de congestionamento. Já os custos de poluição sonora e os custos não calculados de poluição do ar, da água e do aquecimento global, são predominantemente externalidades arcadas por outros agentes da sociedade. 


\begin{tabular}{|l|r|r|r|r|r|r|}
\hline \multicolumn{1}{|c|}{ Tabela 2- Responsabilidade pelos Custos Sociais do Transporte Rodoviário nos EUA, } \\
em 2000 (US\$ 10^6)
\end{tabular}

Nota: Custos para estimativa média. ${ }^{* *}$ não foi possível mensurar na ocasião.

Fonte: Transportation Statistics Annual Report - BTS 1996.

Para se ter uma noção da importância relativa desses custos, em relação aos demais custos rodoviários, estima-se que, em 2000, os EUA tenham gastado cerca de US\$ 125 bilhões em programas de construção, restauração e conservação rodoviária, em todos os níveis de governo.

É necessário ressaltar que há uma variação considerável desses custos quando tratamos dos custos marginais de uma viagem específica, por tipo de veículo, horário, e local da rodovia, para destacar apenas algumas das dimensões mais importantes. A tabela 3, por exemplo, apresenta os custos marginais associados a uma milha adicional de viagem por diferentes classes de veículo, estimados para o estudo destacado acima. Observe-se a ampla variação dos custos de congestionamento, mesmo em termos médios, entre áreas urbanas e rurais, para os diversos tipos de veículo; ou ainda os impactos no pavimento entre os veículos de carga. 


\begin{tabular}{|c|c|c|c|c|c|c|}
\hline \multicolumn{7}{|c|}{$\begin{array}{l}\text { Tabela 3- Custos Marginais de Pavimentação, Congestionamento, Acidentes, Poluição do } \\
\text { Ar e Sonora, para Classes de Veículo e Condições de Tráfego, nos EUA, em } 2000 .\end{array}$} \\
\hline \multirow{2}{*}{ Veículo e Rodovia } & \multicolumn{6}{|c|}{ Custo Marginal (US\$ centavos por milha) } \\
\hline & $\begin{array}{c}\text { Pavi } \\
\text { mento }\end{array}$ & $\begin{array}{r}\text { Congesti } \\
\text { onamento }\end{array}$ & Acidentes & $\begin{array}{l}\text { Poluição } \\
\text { do Ar }\end{array}$ & $\begin{array}{l}\text { Poluição } \\
\text { Sonora }\end{array}$ & Total \\
\hline Autos/Rodo Pav. Rural & 0 & 0.78 & 0.98 & ** & 0.01 & 1.77 \\
\hline Autos/Rodo Urbana & $\overline{0.1}$ & $\overline{7.70}$ & 1.19 & ** & 0.09 & 9.08 \\
\hline $\begin{array}{l}\text { Caminhão } 40 \text { klb } 4 \\
\text { eixos Rod. Rural }\end{array}$ & $\overline{1.0}$ & 2.45 & 0.47 & ** & 0.09 & 9.08 \\
\hline $\begin{array}{l}\text { Caminhão } 40 \text { klb } 4 \\
\text { eixos Caminhão Rod. } \\
\text { Urbana }\end{array}$ & 3.1 & 24.48 & 0.86 & ** & 1.50 & 29.94 \\
\hline $\begin{array}{l}\text { Caminhão } 60 \text { klb } 4 \\
\text { eixos Caminhão Rural } \\
\text { Interstate }\end{array}$ & 5.6 & 3.27 & 0.47 & ** & 0.11 & 9.45 \\
\hline $\begin{array}{l}\text { Caminhão } 60 \text { klb } 4 \\
\text { eixos Caminhão Rod. } \\
\text { Urbana }\end{array}$ & 18.1 & 32.64 & 0.86 & ** & 1.68 & 53.28 \\
\hline $\begin{array}{l}\text { Caminhão } 60 \text { klb } 5 \\
\text { eixos Artic. Rod. Rural }\end{array}$ & $\overline{3.3}$ & $\overline{1.88}$ & 0.88 & ** & 0.17 & 6.23 \\
\hline $\begin{array}{l}\text { Caminhão } 60 \text { klb } 5 \\
\text { eixos Artic. Rod. } \\
\text { Urbana }\end{array}$ & 10.5 & 18.39 & 1.15 & ** & 2.75 & 32.79 \\
\hline $\begin{array}{l}\text { Caminhão } 80 \text { klb } 5 \\
\text { eixos Artic. Rod. Rural }\end{array}$ & 12.7 & 2.23 & 0.88 & ** & 0.19 & 16.00 \\
\hline $\begin{array}{l}\text { Caminhão } 80 \text { klb } 5 \\
\text { eixos Artic. Rod. } \\
\text { Urbana }\end{array}$ & 40.9 & 20.06 & 1.15 & ** & 3.04 & 65.15 \\
\hline \multicolumn{7}{|c|}{$\begin{array}{l}\text { NOTAS: Artic. = Articulado cavalo-carreta; klb = kilo libras; Custos para estimativa média; } \\
* \star \text { não foi possível mensurar na ocasião. }\end{array}$} \\
\hline
\end{tabular}

\section{Mensuração dos Custos da Poluição do Ar}

Veículos automotores produzem emissões que afetam negativamente a qualidade do ar e a saúde de seres humanos e animais. O transporte é um dos maiores causadores de poluição do ar no Brasil e no mundo. No Brasil, o consumo energético de derivados de petróleo e gás natural alcança 110 milhões de toneladas equivalente de petróleo (tep). Os transportes 
consomem $57 \%$ desse total, sendo o transporte rodoviário responsável por $90 \%$ do consumo em transportes.

A poluição do ar é um exemplo emblemático de externalidade, em que os custos são pagos principalmente pelos não usuários de transporte. Algumas regulações governamentais, entretanto, internalizaram alguns desses custos, na forma de preços pagos por veículos novos menos poluentes, regras para renovações anuais de licenças, etc. Nos EUA, por exemplo, a agência ambiental federal - EPA estabeleceu metas de redução de emissões de veículos diesel, a partir de 1980 (tabela 4). No Brasil, há também limites de emissão a serem atendidos por veículos diesel novos, a partir 2002. Em 2012, o país exigirá que os caminhões novos atendam às normas do padrão Euro 5 / Conama 7, que exigem diesel com baixo teor de enxofre e o uso de aditivo à base de uréia para reduzir as emissões de poluentes.

\begin{tabular}{|c|c|c|c|c|}
\hline \multicolumn{5}{|c|}{ Tabela 4- Níveis de Emissão para Veículos Diesel Novos da } \\
EPA (gramas/HP-hora) \\
\hline Ano & HC & CO & NO $_{\mathbf{x}}$ & Partículas \\
\hline 1980 & 1,5 & 25,0 & - & - \\
\hline 1985 & 1,3 & 15,5 & 10,7 & - \\
\hline 1990 & 1,3 & 15,5 & 6,0 & 0,6 \\
\hline 1995 & 1,3 & 15,5 & 5,0 & 0,1 \\
\hline
\end{tabular}

Nota: $\mathrm{HC}=$ hidrocarbonetos; $\mathrm{CO}=$ monóxido de carbono; $\mathrm{NOx}=0$ xidos de nitrogênio

Os impactos causados pelas emissões de veículos variam consideravelmente dependendo das condições meteorológicas, da densidade demográfica, congestionamento do tráfego, usos do solo próximo e outras características situacionais, podendo esta variação atingir várias ordens de grandeza. Os métodos de mensuração do impacto ambiental das emissões podem ser descritos pela equação dada a seguir (Boyer, 1997; pg. 380):

Custo ambiental por tipo de tráfego e veículo = A.B.C.D.E.F, (1) onde

A = quantidade de tráfego de um dado veículo em dadas condições; 
B = emissão unitária, por tipo, gerada por unidade de tráfego;

$\mathrm{C}=$ concentração de poluentes gerada por unidade de emissão, por tipo;

D = nível de exposição dos afetados por unidade de concentração de poluente;

$\mathrm{E}=$ conseqüências do nível de exposição a poluentes;

$\mathrm{F}=$ custo unitário por conseqüência da poluição.

Já o efeito estufa é usualmente custeado através de associações simples de um custo unitário por tonelada de $\mathrm{CO}_{2}$ emitida, variando de US\$ 3 a 125 (DOT, 1998; TRB, 1996), muito embora motores diesel emitam outros gases que contribuem para esse efeito. À taxa de US\$ 3,46 por tonelada de $\mathrm{CO}_{2}$ emitido, o custo equivalente é de US\$ 0,009 por litro consumido de diesel. $^{2}$

As emissões de um veículo diesel dependem de suas dimensões e projeto, tempo de uso, rotação, aceleração e diversos outros fatores. Para os EUA, a agência EPA estimou as emissões médias em condições típicas de uso da frota existente naquele país, em 1994, conforme apresentadas na tabela 5.

\begin{tabular}{|c|c|c|c|c|c|}
\hline \multicolumn{5}{|c|}{ Tabela 5- Emissões de Poluentes por Caminhões Diesel nos EUA (g/mi) } \\
\hline Velocidade & PM10 & NO $_{\mathbf{x}}$ & $\mathbf{C O}$ & VOC & $\mathbf{S O}_{\mathbf{x}}$ \\
\hline 10 & 1,43 & 18,96 & 22,26 & 2,36 & 0,58 \\
\hline 20 & 1,43 & 14,52 & 12,13 & 2,36 & 0,58 \\
\hline 30 & 1,43 & 12,81 & 7,93 & 2,36 & 0,58 \\
\hline 40 & 1,43 & 13,03 & 6,22 & 2,36 & 0,58 \\
\hline 50 & 1,43 & 15,28 & 5,85 & 2,36 & 0,58 \\
\hline 60 & 1,43 & 20,64 & 6,61 & 2,36 & 0,58 \\
\hline
\end{tabular}

Nota: em gramas por milha; extraído de TRB, 1996; pg. 154.

\footnotetext{
${ }^{2}$ Mackenzie et al. (1992; pg. 15) afirmam que, em 1990, os 133 bilhões de galões de diesel e gasolina consumidos nos EUA lançaram 350 milhões de toneladas de dióxido de carbono na atmosfera. Um galão desses combustíveis contém um conteúdo de carbono de cerca de 5,3 libras. (taxas de conversão: (lb) x 0,454 = (kg); (gal) x 3,788 = (litros); (tons) x 0,907 = (toneladas-métricas)
} 
No caso brasileiro, há estimativas da CETESB (1994) para emissões de automóveis e ônibus médios, na a cidade de São Paulo, de CO, HC, NOx e particulados. Esses dados são apresentados em forma de equações relacionadas à velocidade em IPEA/ANTP (2000).

A concentração atmosférica de poluentes depende da taxa de emissão, criação e destruição de poluentes, através de processos atmosféricos físicos e químicos, taxas de transporte para dentro e fora da área em questão, e taxas de sedimentação, entre outros. Estudos mais simples supõem uma relação linear entre a variação proporcional da emissão e a variação proporcional da taxa concentração de poluentes. As concentrações de poluentes, por sua vez, em cada região, dependem de várias fontes de poluição e devem ser medidas ou estimada. O TRB (1996), por exemplo, calcula o nível de concentração por município através do produto de emissões totais per capita, de cada poluente, vezes a população municipal.

A exposição da população aos poluentes é medida em pessoa-ano-micrograma $/ \mathrm{m}^{3}$. Os custos econômicos, por seu turno, são medidos pelo valor monetário associado à morte prematura, sofrimento, doenças e perdas econômicas causadas pela exposição à poluição, medidos em $\$ /$ pessoa-ano/(micrograma $\left./ \mathrm{m}^{3}\right)$. Harrison et. al. (1993) estimam esses custos conforme a tabela 6, abaixo; valores estes compatíveis com um custo da vida humana perdida em acidentes de trânsito entre US\$ 1 e 2 milhões.

\begin{tabular}{|c|c|}
\hline \multicolumn{2}{|c|}{ Tabela 6- Custos de Exposição a Poluentes } \\
\hline Poluente & Custo \\
\hline PM10 & 19,62 \\
\hline NO2 & 0,68 \\
\hline CO & 0 \\
\hline VOC & 0,15 \\
\hline SO2 & 0,31 \\
\hline
\end{tabular}

Nota: custos em US $\$$ pessoa-ano/(micrograma $\left./ \mathrm{m}^{3}\right)$; $\mathrm{COV}=$ compostos orgânicos voláteis. Fonte: Harrison et. al. (1993) e TRB (1996). 
O estudo do IPEA/ANTP (2001) utiliza valores médios dos impactos de emissões, expressos em US\$/kg, transformados em reais e ajustados de forma a expressar a diferença entre as rendas per capita brasileira e americana. ${ }^{3}$

Nos estudos de caso do TRB (1996), o cálculo do custo de exposição causado por um grama adicional emitido de poluente segue a seguinte metodologia de forma simplificada.

$$
\begin{aligned}
& Y_{z}=C_{z} \cdot p_{z} \cdot P / E_{z} \quad \text { (2) onde } \\
& Y_{z}=\text { custo de exposição causado por um grama adicional emitido de poluente } z ; \\
& C_{z}=\text { concentração média anual do poluente } \mathrm{z} \text { na região }\left(\text { micrograma } / \mathrm{m}^{3}\right) ; \\
& p_{z}=\text { custo unitário de exposição ao poluente } \mathrm{z}\left(\$ / \text { pessoa-ano-micrograma } / \mathrm{m}^{3}\right) ; \\
& P=\text { população da região; } \\
& E_{z}=\text { emissão primária do poluente } \mathrm{z} \text { por todas as fontes numa região }(\mathrm{g} / \mathrm{ano}) \text {. }
\end{aligned}
$$

Como os modelos de emissão primária de poluentes utilizados são linearmente proporcionais à população, a fórmula acima pode ser reduzida a:

$$
\begin{aligned}
& \mathrm{Y}_{\mathrm{z}}=\mathrm{C}_{\mathrm{z}} \cdot \mathrm{p}_{\mathrm{z}} / \mathrm{k}_{\mathrm{z}} \quad \text { (3) onde } \\
& \mathrm{k}_{\mathrm{z}}=\text { constante específica para o poluente } \mathrm{z} \text {. }
\end{aligned}
$$

No caso do PM10, $\mathrm{k}_{\mathrm{z}}$ situa-se entre 0,11 e 0,15 x $10^{6}$. Tomando o limite inferior como referência, o custo seria $\mathrm{Y}_{\mathrm{z}}=\mathrm{US} \$ 0,00535 /$ grama. Tomando o nível de emissão marginal de PM10 caminhões da tabela 5 (1,43 gramas/milha), tem-se um custo de US\$ 0,007652/milha percorrida para uma situação de rodovia rural.

Os estudos de caso do TRB (1996) apresentam custos unitários médios de poluição, para todos os poluentes relevantes conforme apresentado na tabela 7 .

\footnotetext{
${ }^{3}$ Os valores adotados foram: $\mathrm{CO}=\mathrm{R} \$ 0,19 / \mathrm{kg} ; \mathrm{HC}=\mathrm{R} \$ 1,14 / \mathrm{kg} ; \mathrm{NOx}=\mathrm{R} \$ 1,12 / \mathrm{kg} ;$ Partículas=R $\$ 0,91 / \mathrm{kg}$ de emissão.
} 


\begin{tabular}{|c|c|c|c|c|}
\hline \multicolumn{5}{|c|}{ Tabela 7- Custos de Poluição por Viagem e Unitário (todos os poluentes) } \\
\hline & & Localização da Rodovia & \\
\hline Caso & & Rural & Urbana & Total \\
\hline 1A & Extensão da rodovia (milha) & 321 & 29 & 350 \\
\hline 1A & Custo da Poluição (US\$) & 5,94 & 0,60 & 6,54 \\
\hline 1A & Custo unitário (US\$/mi) & 0,0185 & 0,0207 & 0,0187 \\
\hline 3A & Extensão da rodovia (milha) & 3237 & 512 & 3749 \\
\hline 3A & Custo da Poluição (US\$) & 48,5 & 15,15 & 63,65 \\
\hline 3A & Custo unitário (US\$/mi) & 0,0157 & 0,0296 & 0,0170 \\
\hline
\end{tabular}

Fonte: TRB (1996; pg. 90 e 95). Caso 1A carregamento de grão de Walnut Grove, Minesota para o porto de Winona, no rio Mississippi; caso 3A carregamento de contêiner de Long Beach, CA., para Chicago.

No caso do cálculo dos custos associados à poluição do ar, uma sugestão metodológica é utilizar a fórmula simplificada para o custo marginal dada em (3). Nesse caso, os dados básicos necessários seriam a concentração de poluição, por poluente e região (distrito censitário, município ou microrregião) e os custos de exposição aos poluentes que seriam adaptados das fontes anteriormente citadas e de Motta (1994). Poderiam ser mantidas as constantes do estudo do TRB, assim como os níveis de emissão de poluentes de caminhões (números conservadores).

\section{Mensuração dos Custos da Poluição Sonora}

Os custos da poluição sonora são usualmente estimados através do cálculo da redução do valor de propriedades causados por ruído recorrente de veículos. ${ }^{4}$ Medidas de níveis de ruído emitidos e captados a distâncias específicas são associados aos valores das propriedades, permitindo medir seus impactos específicos. A tabela 8 apresenta a amplitude de variação do preço de propriedades, em termos percentuais, por cada decibel de ruído acima de um limite inferior.

\footnotetext{
${ }^{4}$ Paara o caso do modal aéreo ver Bastos et. al., 2007.
} 


\begin{tabular}{|c|c|c|c|}
\hline \multicolumn{4}{|c|}{ Tabela 8- Impacto da Poluição Sonora nas Propriedades } \\
\hline & Alto & Médio & Baixo \\
\hline Variação \% do valor por decibel & 0,88 & 0,40 & 0,14 \\
\hline
\end{tabular}

Fonte: DOT, 1998.

Já o TRB (1996) utiliza valores médios por milha trafegada por caminhões, por localização da rodovia (US\$ 0,020/mi em rodovias expressas urbanas; US\$ 0,065 em rodovias urbanas em geral; e zero em rodovias rurais). Esses valores são compatíveis com fontes que estimam o valor do impacto percentual no preço de propriedades de $0,40 \%$ por decibel acima de um limite inferior.

Uma sugestão para o cálculo desse impacto, no caso brasileiro, seria aplicar os menores valores unitários acima como limite superior desse impacto, testando, alternativamente, valores inferiores.

\section{Mensuração dos Custos de Acidentes}

Conforme visto na seção 1 , os custos de acidentes dominaram os componentes dos custos sociais nos EUA. No caso brasileiro, é de se esperar que o mesmo ocorra, tendo em vista o número proporcionalmente maior de acidentes em nossas estradas. Somente nos 37.000 quilômetros de estradas federais sob responsabilidade da Polícia Rodoviária Federal, em 2007, foram reportados 128.476 acidentes, com 7.228 vítimas fatais, 24.202 vítimas com lesões graves, e 58.330 lesões leves.

Como no caso de congestionamento, parte dos custos dos acidentes é suportado pelos próprios envolvidos, ou seja, são custos internos. Outra parte, porém, é suportada por outros, como no caso de atropelamento de pedestres e ciclistas, e particularmente no aumento do risco de acidentes com o aumento do tráfego de veículos.

Dessa forma, uma metodologia que permitiria calcular o custo total e marginal dos acidentes envolveria os seguintes passos: 
a) estimar relações entre o volume de tráfego e a freqüência de acidentes por tipo (somente caminhão; caminhão-caminhão; caminhão-auto; caminhão-ônibus; autoauto; outros) e por intensidade (com vítimas fatais; com vítimas com lesões graves; com vítimas com lesões leves; sem vítimas);

b) com base nessas relações, calcular a variação esperada no número de acidentes, por categoria, pelo tráfego incremental de um veículo;

c) avaliar esses incrementos monetariamente, com base nos custos imputados a cada categoria de acidentes;

d) estimar qual parte desses custos é interna (paga pelo usuário operador) e qual é externa.

Dessa forma, o conjunto de dados mínimo para se aferir esse item de custo demandaria informações detalhadas de acidentes, por tipo, por rodovia (e suas características, especialmente volume de tráfego), e indicações dos custos associados (e o mesmo para o caso de ferrovias).

Outro aspecto crítico desse item é a imputação do valor atribuído à vida humana ou às lesões oriundas de acidentes. Nos EUA, o assunto foi revisitado por Miller et al. (1991), concentrando-se nos estudos que procuraram medir a disposição de pagar de indivíduos por equipamentos de segurança que reduzem o risco de acidentes e morte. Nesse caso, o custo total de um acidente, por vítima fatal, foi estimado em US\$2,3 milhões (incluindo o valor imputado à vida humana, danos materiais e de eventual congestionamento na rodovia), e o custo por lesão em US\$ 46 mil. Para o caso brasileiro, há estimativas de Motta (1994) e de indenizações pagas pelo INSS relativas a acidentes no setor de transportes.

\section{Mensuração dos Custos de Congestionamento}

Os custos de congestionamento incluem (DOT, 1998):

a) tempo adicional de viagem de pessoas e veículos comerciais;

b) a variação do consumo de combustível e outros custos operacionais pela redução de velocidade; 
c) o aumento da variabilidade do tempo de viagem;

d) o aumento do desgaste de motoristas e passageiros.

O impacto dos diversos tipos de veículos no congestionamento é usualmente uniformizado através de medidas de equivalência. O Highway Capacity Manual (TRB, 1994) mede a contribuição de caminhões ao congestionamento em unidades equivalentes de automóveis (PCE). Um caminhão com um PCE igual a três teria o mesmo efeito em termos de congestionamento que três automóveis. Os valores dos PCE dependem do peso bruto, potência, comprimento e dirigibilidade dos veículos; variam também consideravelmente dependendo do tipo de rodovia, sendo o perfil vertical particularmente importante para se determinar o PCE de caminhões pesados, que operam em velocidades menores em subidas íngremes e longas (DOT, 1998).

O modelo clássico da teoria econômica do congestionamento indica a direção da causalidade: a densidade de tráfego determina a velocidade; quando um veículo adicional entra na rodovia, a densidade aumenta, a velocidade cai e o tempo de viagem (a recíproca da velocidade) aumenta. As condições de tráfego e velocidade são geralmente expressas através de curvas de velocidade versus volume, que podem ser convertidas para curvas de tempo de viagem versus volume. Usando um valor representativo do custo do tempo para um motorista representativo, essa última curva pode ser convertida em uma relação de custo variável médio por veículo equivalente - AVC (Hau, 1992). A derivação do custo marginal do veículo adicional é então dada por (Walters, 1961; Hau, 1992):

$$
\begin{aligned}
\mathrm{MC} & =\partial \mathrm{C}(\mathrm{Q}, \mathrm{K}) / \partial \mathrm{Q}=\partial(\mathrm{Q} \cdot \operatorname{AVC}(\mathrm{Q}, \mathrm{K})+\mathrm{F}) / \partial \mathrm{Q}=\operatorname{AVC}(\mathrm{Q}, \mathrm{K})+\mathrm{Q} \cdot \partial \mathrm{AVC}(\mathrm{Q}) / \partial \mathrm{Q}= \\
& =\operatorname{AVC}(\mathrm{Q}, \mathrm{K})(1+\varepsilon) .
\end{aligned}
$$

O custo marginal é dado então por um componente de custo variável médio AVC, sentido por

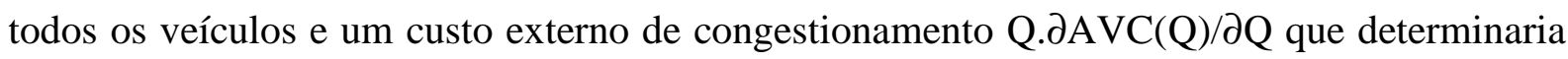
a taxa ótima de congestionamento. $\varepsilon$ é a elasticidade da curva AVC. Note-se que AVC depende da densidade de tráfego $\mathrm{Q}$ e da capacidade da rodovia $\mathrm{K}$.

Há ainda dois conjuntos de informações relevantes para o caso em tela. O primeiro é a variação do volume de tráfego ao longo do dia e a participação do tráfego de carga nesse 
tráfego. De maneira geral, pode-se dizer que nas horas de picos há um aumento considerável da participação de automóveis no tráfego total. No mesmo sentido, veículos de carga podem evitar mais facilmente horários de pico previsíveis.

O segundo aspecto a quantificar é a ocorrência de congestionamentos não recorrentes, principalmente devidos a acidentes, e que podem causar aumentos significativos nos tempos de viagem. Tendo em vista a grande participação de caminhões na ocorrência de acidentes, no Brasil, esse componente do custo de congestionamento pode ser significativo.

A tabela 9 resume os itens de dados necessários à mensuração dos custos de congestionamento, possíveis fontes e tratamentos.

\begin{tabular}{|l|c|c|c|}
\hline \multicolumn{3}{|c|}{ Tabela 9- Dados para a Mensuração dos Custos de Congestionamento } \\
\hline \multicolumn{1}{|c|}{ Item } & Fontes Possíveis & Discriminação & Tratamento \\
\hline Curvas velocidade-volume de tráfego & HCM; DNIT & $\begin{array}{c}\text { Por tipo de } \\
\text { rodovia }\end{array}$ & $\begin{array}{c}\text { Adaptação do } \\
\text { HCM }\end{array}$ \\
\hline Valor representativo do tempo & TRB; IPEA/ANTP & Tipo de usuário & $\begin{array}{c}\text { Adaptação do } \\
\text { TRB }\end{array}$ \\
\hline $\begin{array}{l}\text { Variação dos volumes de tráfego } \\
\text { diário }\end{array}$ & DNIT; DERs & Tipo de rodovia & \\
\hline $\begin{array}{l}\text { Variação dos volumes de tráfego } \\
\text { diário de caminhões }\end{array}$ & DNIT; DERs & Tipo de rodovia & \\
\hline $\begin{array}{l}\text { Acidentes com part. de caminhões e } \\
\text { congestionamentos }\end{array}$ & DNIT; DERs & Detalhamento & \\
\hline
\end{tabular}

\section{Mensuração do Custo de Infraestrutura}

Os impactos do tráfego sobre o pavimento de uma estrada resultam das várias forças transmitidas através dos pneus dos veículos. Dentre tais forças, capazes de deteriorar os pavimentos, são particularmente importantes as forças verticais transmitidas pela superfície de contato dos pneus com o pavimento, afetando tanto as camadas superficiais do revestimento quanto as suas camadas estruturais mais profundas. São essas forças que são consideradas nas normas que norteiam o dimensionamento de pavimentos. 
Para a avaliação do poder de deterioração dos pavimentos por essas forças foram desenvolvidos métodos práticos que permitem determinar o poder de destruição das várias configurações de veículos e de tipos de eixos, tendo-se como base o poder de destruição medido para um eixo padrão de 8,2 t. Resultou disso o conceito do Fator de Equivalência de Carga (FEC ou ESAL), que expressa o número necessário de repetições (passagens) de uma solicitação (carga) de um determinado tipo de eixo que provoca a mesma deterioração no pavimento provocada pela solicitação padrão (eixo padrão de $8.165 \mathrm{~kg}=18.000 \mathrm{lb}$ ).

Os métodos mais largamente utilizados no Brasil para a determinação do FEC são os desenvolvidos pela AASHO - American Association of State Highway Officials e pela USACE - United States Army Corps of Engineers. O DNIT baseou-se nesse último método para a determinação de suas relações de fatores de equivalência de carga.

As equações que definem as equivalências das solicitações práticas em relação ao eixo padrão são as seguintes:

\section{AASHO}

$$
\begin{aligned}
& E D=\left(1,0513 / 8,165^{*} w\right)^{4,32} \\
& E 1=(w / 8,165)^{4,32} \\
& E 2=(w / 15,08)^{4,14} \\
& E 3=(w / 22,95)^{4,22}
\end{aligned}
$$

\section{USACE}

$$
\begin{aligned}
& E D=2,0782 * 10^{-4 *} w^{4,0175} \\
& E 1=1,8320 * 10^{-6} * w^{6,2542} \\
& E 2=1,592 * 10^{-4 *} w^{3,472} \quad(\text { de } 0 \text { a } 11 t) \\
& E 2=1,528 * 10^{-6} * w^{5,484} \quad(>11 t) \\
& \left.E 3=8,0359 * 10^{-5} * w^{3,3549} \quad \text { (de } 0 \text { a } 18 t\right)
\end{aligned}
$$




$$
E 3=1,3229 * 10^{-7} * w^{5,5789} \quad(>18 t)
$$

onde $\mathrm{ED}=$ eixo dianteiro; $\mathrm{E} 1$ = eixo traseiro simples; $\mathrm{E} 2$ = eixo tandem duplo; e E3 = eixo tandem triplo.

Calculando-se os valores de FEC pelos métodos AASHO e USACE, adotando-se como cargas máximas por eixo aquelas determinadas pela legislação brasileira, ${ }^{5}$ conclui-se que:

- são os veículos pesados, ônibus e caminhões, que respondem pela deterioração dos pavimentos das rodovias;

- os dois métodos produzem resultados substancialmente diferentes ao atribuir poder de deterioração dos pavimentos aos eixos tandem duplos e triplos.

Convertidos em FEC, o impacto marginal dos eixos sobre o pavimento é estimado adotandose relações que estabelecem o número de passagens do eixo padrão necessárias para levar o pavimento a um dado nível de deterioração, medido por um índice de rugosidade (pavimento de um dado tipo, espessura, condições de solo e meio ambiente). Uma vez estabelecido um padrão de serviço da rodovia, através de um nível limite de rugosidade, esta, ao atingi-lo, seria recapeada, ganhando nova vida útil para servir ao tráfego por novo período (TRB, 1990; Small et al., 1989). Supondo que o custo de recapeamento seja insensível a pequenas variações de espessura e que esse custo seja constante, por faixa, para os vários tipos de rodovias pavimentadas, o TRB (1996) calcula o valor presente dos custos de todos os recapeamentos futuros como:

$$
\mathrm{C}=\mathrm{c}\left[1 /(1+\mathrm{i})^{\mathrm{Q} / \mathrm{A}+\mathrm{E})}\right] \cdot\left\{1 /\left[(1+\mathrm{i})^{\mathrm{L}}-1\right]\right\}
$$

e o incremento em C para um FEC-km adicional como:

$$
-\mathrm{dC} / \mathrm{dQ} \sim=\mathrm{i} . \mathrm{C} /(\mathrm{A}+\mathrm{E})
$$

onde

$\mathrm{A}=$ número anual de FEC;

\footnotetext{
${ }^{5} 6 \mathrm{t}$ para o eixo dianteiro, $10 \mathrm{t}$ para o eixo traseiro simples, $17 \mathrm{t}$ para o eixo duplo em tandem e $25,5 \mathrm{t}$ para o eixo triplo em tandem.
} 


$$
\begin{aligned}
& \mathrm{E}=\text { desgaste anual do pavimento, medido em FEC, independentemente do tráfego }{ }^{6} \\
& \mathrm{Q}=\text { vida útil remanescente do pavimento medida em FEC; } \\
& \mathrm{L}=\text { intervalo de anos entre recapeamentos; } \\
& \mathrm{c}=\text { custo por km (ou milha) de recapeamento; e } \\
& \mathrm{i}=\text { taxa de juro real. }
\end{aligned}
$$

O valor de Q é tomado como sendo a metade da vida útil de dimensionamento do pavimento. Os demais valores utilizados no estudo são apresentados na tabela 10.

\begin{tabular}{|c|c|c|c|c|}
\hline \multicolumn{5}{|c|}{ Tabela 10- Parâmetros do TRB para o Cálculo do Custo Marginal de Infra-estrutura } \\
\hline Parâmetros do Custo Marginal & Custos de Recapeamento \\
\hline & Valor & & Rural & Urbano \\
\hline Taxa de desconto & $5 \%$ & Vias expressas & 105.000 & 115.000 \\
\hline Vida de projeto do pavimento & 7 anos & Arteriais & 94.000 & 103.000 \\
\hline Desgaste indep. anual do pav. & 100.000 & Outras & 77.000 & 93.000 \\
\hline
\end{tabular}

Fonte: TRB, 1996; custos em US\$/milha de faixa de rolamento corrigidos para US\$ de 1992.

O TRB (1996) ainda imputa ao veículo de carga um custo de US\$ 0,01 relativo à fadiga de pontes (metálicas), por cada passagem carregado, e por cada ponte ao longo do percurso. Para tanto, estima-se o número médio de pontes por quilômetro, por tipo de rodovia, para os estados percorridos nas rotas de interesse.

\section{Conclusões}

A questão do desenvolvimento sustentável ainda é um conceito amplo que vem sendo desafiado pelas demandas da sociedade de transporte e crescente mobilidade. O setor ainda é, sem dúvida, um voraz consumidor de recursos não renováveis no processo de atendimento a esses anseios. O esgotamento desse percurso, no entanto, já vem sendo anunciado, e a sociedade exige meios para redirecionar esse processo de forma eficiente e equitativa.

\footnotetext{
${ }^{6} \mathrm{O}$ parâmetro E também pode ser visto como um limite inferior para a durabilidade do pavimento construído
} 
Nesse sentido, a resposta a questões fundamentais, tais como onde investir, o que regular, ou que preços cobrar, demandam conhecimento aprofundado dos processos produtivos de transporte e seus efeitos sobre os agentes da sociedade. Este artigo buscou revisitar métodos úteis para medir esses efeitos e possibilitar, através de mecanismos de preços, entre outros, internalizar os custos externos dos transportes. A complexidade dessa mensuração é evidente; mais ainda a dificuldade de aceitação social da incorparação desses custos externos nos preços. Porém, a compreensão plena desses impactos é um elemento essencial para permitir alcançar soluções equilibradas e sustentáveis no longo prazo.

\section{Referências}

Bastos, A. L. D., Baum, D. M., e Dias, D. P. (2007), "Ruídos e emissões no transporte aéreo", Revista Literatura dos Transportes, vol 1, n. 1, pp 66-104.

Boyer, K. (1997), Principles of Transportation Economics, Addison-Wesley, N.Y.

CETESB (1994), Relatório das Condições Ambientais em São Paulo.

DOT (1998), Highway Cost Allocation Study Final Report, FHWA/DOT, Wash., D.C.

Harrison, D. et al. (1993), Final Report: External Costs of Electric Utilities Resource Selection in Nevada, National Economic Research Associates, Mass.

Hau, T. (1992), "Economic fundamentals of road pricing”, World Bank Working Paper WPS 1070, Wash. D.C.

IPEA (2000), "Redução das Deseconomias Urbanas pela Melhoria do Transporte Público", Relatório Final. IPEA/ANTP.

Mackenzie et al. (1992), The Going Rate: What it Really Costs to Drive, World Resources Institute, Wash. D.C.

Miller, T. et al. (1991), The Costs of Highway Crashes, DOT/FWHA, oct.

Motta, R. e Ana P. Fernandes (1994), "Custos de saúde associados à poluição do ar no Brasil”, Texto para Discussão no. 332, IPEA.

Santos, E. M. (2010), “The economics of regulating road transport", Revista transportes (resenha).

Small, K. et al. (1989), Road Work: A New Highway Pricing and Investment Policy, The Brookings Institution, Wash., D.C.

TRB (1994), Special Report 209: Highway Capacity Manual, $3^{\text {rd }}$ ed. National Research Council, Wash. D.C.

TRB (1996), Special Report 246: Paying our Way: Estimating Marginal Social Costs of Freight Transportation, National Research Council, Wash. D.C.

Walters, A. (1961), "The theory and measurement of private and social cost of highway congestion", Econometrica, vol. 29(4), oct.

independentemente do volume de tráfego. 